Research Article

\title{
Friction Characteristics of Post-Tensioned Tendons of Full-Scale Structures Based on Site Tests
}

\author{
Haoyun Yuan (D), Yuan Li (iD, Bin Zhou, Shuanhai He, and Peizhi Wang \\ School of Highway, Chang'an University, Xi'an 710064, China \\ Correspondence should be addressed to Yuan Li; liyuan@chd.edu.cn
}

Received 5 September 2019; Revised 12 December 2019; Accepted 27 December 2019; Published 18 April 2020

Academic Editor: Rosario Montuori

Copyright (C) 2020 Haoyun Yuan et al. This is an open access article distributed under the Creative Commons Attribution License, which permits unrestricted use, distribution, and reproduction in any medium, provided the original work is properly cited.

In the design of prestressing concrete structures, the friction characteristics between strands and channels have an important influence on the distribution of prestressing force, which can be considered comprehensively by curvature and swing friction coefficients. However, the proposed friction coefficient varies widely and may lead to an inaccurate prestress estimation. In this study, four full-scale field specimens were established to measure the friction loss of prestressing tendons with electromagnetic sensors and anchor cable dynamometers to evaluate the friction coefficient. The least square method and Bayesian quantile regression method were adopted to calculate the friction coefficient, and the results were compared with that in the specifications. Field test results showed that Bayesian quantile regression method was more effective and significant in the estimation of the friction coefficient.

\section{Introduction}

Prestressed concrete is used to reduce or offset the tensile stress of concrete under the action of loads, so as to improve the crack resistance and stiffness of components. Prestressed distribution plays an important role in the design and construction of prestressed concrete structures. For posttensioned concrete structures, the friction characteristics between the steel strand and the pipe (also known as sheaths) are directly related to the elongation required by the tensioning operation and the prestress force distribution. The value of friction coefficient is usually obtained from the design specifications or the instructions provided by manufacturers. The recommended friction coefficient [1-4] ranges widely, which may result in the confusion and inaccuracy. The inaccurate calculation of friction loss can cause unreasonable camber and prestress reduction or even deflection of beams. Aiming to obtain the true force of the structure, many researches have been carried out to investigate by the calculation method [5] and the full-scale field tests [6-8]. When the friction loss data of prestress is obtained by tests, the corresponding friction coefficient can be got according to the prediction equation of prestress. In addition, the post-tensioning method cannot guarantee the accuracy of stress measurements of steel strands $[9,10]$. The total prestress loss at the beam end and the stress distribution are measured by the pressure sensor at the anchorage point [11] and the electromagnetic (EM) sensor [12-14], respectively.

However, it is possible for the designers and builders to determine the coefficients erroneously. Although many studies have been conducted to determine the friction coefficient by various methods, no standard calculation method has been established [15-17]. The least square method [18] is the most common calculation method for estimating the friction coefficient. First and foremost, such method needs to assume that the random error term obeys the zero mean and homoscedastic distribution. Secondly, it is essentially based on the conditional mean model, mainly in the statistical analysis framework of the mean values. Nevertheless, too much pursuit of the average gives rise to the loss of much other information. Furthermore, the field test environment is less controllable than the precision laboratory, and the data are more discrete. Considering the cost factor, the sample size of the field test is often small, so the accuracy of the results estimated by the least square method is low. 
In this study, a Bayesian quantile estimation method $[19,20]$ was proposed in order to make up for the deficiency of the least square method in the calculation of the friction coefficient. The Bayesian quantile regression method does not require mandatory distribution of test data, and the friction coefficient can still be effectively estimated even when the sample size is small. Based on the monitoring of the prestressed tension process of four full-scale test beams in the construction site, the above two calculation methods of friction coefficient were compared.

\section{Friction Coefficients in Post- Tensioning System}

During the tensioning process of the steel strand, the contact between the steel strand and the pipe can cause two types of friction loss: the curvature friction that resulted from the curved part of the pipe and the wobble friction due to the deformation of the pipe.

The regression analysis model $[1,21]$ of prestressing friction loss parameters is presented as

$$
\Delta f_{p F}=f_{p j}\left(1-e^{-(k x+\mu \theta)}\right),
$$

where $f_{p j}$ is the stress in the prestressing steel at jacking; $x$ is length of a prestressing tendon from the jacking end to any point under consideration; $k$ is wobble friction coefficient; $\mu$ is coefficient of friction; $\theta$ is sum of the absolute values of angular change of prestressing steel path from jacking end, or from the nearest jacking end if tensioning is done equally at both ends, to the point under investigation (rad.); and $e$ is the base of Napierian logarithms.

Taking logarithms on both sides of an equal sign equation (1), the equation can be derived as

$$
k x+\mu \theta=\ln \left(\frac{f_{p j}}{f_{p j}-\Delta f_{p F}}\right) .
$$

Let

$$
Y=\ln \left(\frac{f_{p j}}{f_{p j}-\Delta f_{p F}}\right) .
$$

The equation can be presented as

$$
Y=k x+\mu \theta+\varepsilon
$$

Assuming that $x$ and $\theta$ are deterministic variables, $Y$ is random variables, $k$ and $\mu$ are constants, and $\varepsilon$ is the error term, then equation (4) can be regarded as a binary linear regression model.

Table 1 lists the friction coefficients recommended for strands in galvanized metal pipes in various provisions, which is the most widely used type of pipes. It can be found that various provisions give different ranges, some of which are ranges while others are fixed values. In general, unpractical calculation of friction loss can lead to unreasonable buckling and reduction of prestressing. Overestimating the value of friction loss may lead to excessive prestressing, which will bring about excessive buckling and shrinkage of members. This study attempted to estimate the coefficient of friction based on Bayesian quantiles and verify the current practice, shown in Table 1.

\section{Methodologies to Derive Friction Coefficients}

Using EM sensors to obtain the prestress distribution of the tendon, the friction coefficient can be derived as follows.

To start with, the differences between the anchor point and other measurement points are substituted into equation (4), which are overconditioned simultaneous equations because $k$ and $\mu$ are unknown; a number of equations can be formed. Therefore, the unique coefficient usually cannot be obtained in this way, and a statistical approach is required instead. In an attempt to solve this problem, two equations were chosen among many equations as shown in equation (5), and then $k$ and $\mu$ were calculated. Choosing another set of two equations may cause $k$ and $\mu$ to be slightly different. Then, the average coefficients of different simultaneous equations are used as the friction coefficients:

$$
\left[\begin{array}{ll}
x_{1} & \theta_{1} \\
x_{2} & \theta_{2}
\end{array}\right]\left[\begin{array}{l}
k \\
\mu
\end{array}\right]=\left[\begin{array}{l}
Y_{1} \\
Y_{2}
\end{array}\right] .
$$

The least square method can be adopted. By modifying equation (4), the sum of squares can be formed as displayed in equation (6), including each possible combination of two arbitrary points. Then, the minimum two coefficients of this equation can be regarded as $k$ and $\mu$ :

$$
\min \sum_{i=1}^{n}\left(Y_{i}-k i x i+\mu i \theta i\right)^{2} .
$$

However, the application of least square method analysis has some limitations. First of all, it is necessary to assume that the random error term is independent and obeys the distribution of zero mean and same variance, which is often difficult to meet the requirements of field test data. Besides, it is essentially based on the conditional mean model, mainly in the framework of the mean statistical analysis, ignoring the information of the distribution tail. The result of being too mean is to lose a lot of other information. Hence, Bayesian quantile regression method $[19,20]$ can be adopted to estimate the parameters $k$ and $\mu$.

3.1. Quantile Regression. Since quantile regression was proposed [23], its theoretical research and practical application have been developed rapidly [24-27]. The calculation model is based on a combined conditional quantile function model. It can characterize the influence of the quantiles of the dependent variable, which covers for the shortcomings of only focusing on the mean value.

For a given $x_{i}$, condition $\mathrm{P}$ quantile of $y_{i}$ is

$$
q_{p}\left(y_{i} \mid x_{i}\right)=x_{i}^{\prime} \beta,
$$

where $\beta(p)$ is a coefficient vector that only depends on $\mathrm{P}$. The P-regression quantile can be defined as an arbitrary solution of the quantile regression minimization lower equation problem, which is presented as 
TABle 1: Recommended friction coefficients.

\begin{tabular}{lcc}
\hline Provisions & Provisions wobble friction coefficient $(k) / \mathrm{m}$ & $\begin{array}{c}\text { Curvature friction coefficient } \\
(\mu) / \text { rad }\end{array}$ \\
\hline ACI 318-08 [21] & $0.0016-0.0066$ & $0.15-0.25$ \\
ACI 318-14 [2] & Not specified but can refer to post-tensioning \\
AASHTO LRFD bridge design specifications [3] & 0.00066 & $0.15-0.25$ \\
Bridge design manual [22] & 0.00066 & 0.2 \\
Post-tensioning manual [4] & $0.0010-0.0023$ with 0.0016 recommended & $0.14-0.22$ with 0.18 recommended \\
JTG 3362-2018 [1] & 0.0015 & $0.20-0.25$ \\
\hline
\end{tabular}

$$
\min _{\beta} \sum_{i} \rho_{p}\left(y_{i}-x_{i}^{\prime} \beta\right)
$$

where

$$
\rho_{p}(u)=u(p-I(u<0)) .
$$

Equation (9) is defined as loss function, as known as "kernel function".

3.2. Asymmetric Laplace Distribution (ALD). Research results illustrate that the traditional symmetric Laplace laws are a subset of ALD [28]. The application of ALD in quantile regression has been supplemented and improved in theory [20, 28-30].

If a random variable $Y_{\sigma, \mu}$ is considered to obey an asymmetric Laplacian distribution, it should satisfy the following conditions; the position parameter $\mu \in R$, the scale parameter $\sigma>0$, and the characteristic function has the following form:

$$
\psi(\mathrm{t})=\left[1+\sigma^{2} t^{2}-i \mu t\right]^{-1}
$$

and its distribution is followed as $Y_{\sigma, \mu} \sim A L D(\sigma, \mu)$.

Maximum likelihood estimation is one of the most popular methods in statistical inference. For equation (4), assuming that the error of the model obeys normal distribution and the mean value is 0 , the standard deviation $\sigma$ is known, i.e., $\varepsilon \sim N\left(0, \sigma^{2}\right)$, the likelihood function based on parameter $\beta$ of sample $\left(x_{i}, y_{i}\right)(i=1,2 \cdots n)$ is as follows:

$$
L(\beta) \propto \exp \left\{-\frac{1}{2 \sigma^{2}} \sum_{i=1}^{n}\left(y_{i}-x_{i}^{T} \beta\right)^{2}\right\} .
$$

Maximization of likelihood function $L(\beta)$ can be achieved by least square estimation. If the probability density function of the error term $\mathcal{E}$ of the model is assumed to be

$$
f(\varepsilon) \propto \exp \left\{-\sum_{i=1}^{n} \rho_{p}\left(y_{i}-x_{i}^{T} \beta\right)\right\},
$$

where $\rho_{p}(\bullet)$ is the loss function. Based on this, considering the asymmetric Laplace's standard density function, the form is as follows:

$$
f(\varepsilon)=p(1-p) \exp \left\{-\rho_{p}(\varepsilon)\right\} .
$$

Minimizing the loss function equation (8) is equivalent to maximizing the likelihood function of the asymmetric
Laplacian density of the independent joint distribution. If the random variable $\mu$ conforms to an asymmetric Laplace distribution, its probability density satisfies the formula as follows:

$$
f(\mu)=p(1-p) \exp \left\{-\rho_{p}(\mu)\right\} .
$$

3.3. Bayesian Quantile Regression. At present, a very common phenomenon is that Bayesian statistical inference is applied to quantile regression [19, 20, 29-31]. Scholars [32-35] have developed it into a systematic theory and method of statistical inference and applied it in various fields $[36,37]$. Bayesian analysis method introduces the prior information of relevant parameters to improve the effect of statistical inference. And the posterior distribution of the parameters obtained is compared with the parameters estimated by the traditional quantile regression regarding parameters as random variables.

Assuming that the model error obeys an asymmetric Laplace distribution, the basic idea of Bayesian quantile regression is that the posterior density of the parameters is finally obtained by (1) transforming the problem of minimizing the loss function into a likelihood function that maximizes the asymmetric Laplace density of the independent joint distribution of the error terms and (2) assuming the prior distribution of the parameters and adopting the appropriate sampling algorithm.

If conditional quantiles are to be computed, no matter what the initial distribution of data is, two assumptions need to be satisfied in the generalized linear model:

(1) $f\left(\mathrm{y} ; \mu_{i}\right)$ is asymmetric Laplace density.

(2) For any $0<p<1$, the connection function is $g\left(\mu_{i}\right)=x_{i}^{\prime} \beta(p)=q_{p}\left(y_{i} \mid x_{i}\right)$. For a given observation value $y=\left(y_{1} \ldots y_{n}\right)$, the $\beta$ posterior distribution $p(\beta \mid y)$ can be presented as

$$
p(\beta \mid y) \propto L(\beta \mid y) p(\beta),
$$

where $p(\beta \mid y)$ is a prior distribution of $\beta, L(\beta \mid y)$ is a likelihood function, which can be written by the Bayesian formula and a position parameter $\mu_{i}=x_{i}^{\prime} \beta(p)$ :

$$
L(\beta \mid y)=p^{n}(\beta)(1-p(\beta))^{n} \exp \left\{-\sum_{i} \rho_{\mathrm{p}}\left(\mathrm{y}_{i}-x_{i}^{\prime} \beta\right)\right\} \text {. }
$$


Any prior distribution can be applied to equation (16), and Bayesian quantile regression transforms the solution of the original model into the solution of the likelihood function of equation (16).

3.4. MCMC Method. The advent of Markov chain Monte Carlo (MCMC) methods [38-40] largely solves the problem of computing the high dimensional integrals, which is necessary to characterize the posterior distributions for most models. To begin with, a Markov chain is constructed by sampling. The limiting distribution of the Markov chain is the actual distribution $f(\theta)$ of a parameter $\theta$. With the convergent Markov chain, parameters of the quantile model are then estimated. The Gibbs sampling, which is one of the most popular MCMC algorithms in the Bayesian analysis $[41,42]$, is adopted herein to facilitate the calculation.

The parameter estimation process is considered by the principle of Gibbs sampling algorithm, and the estimated parameters are $k$ and $\mu$. Let $X$ represent the available data set and $M$ represent the accepted model, supposing that the following two conditional distributions are known as $f_{1}(k \mid \mu, \mathrm{X}, \mathrm{M})$ and $f_{2}(\mu \mid k, \mathrm{X}, \mathrm{M})$, where $X$ is obtained from full-scale field tests.

Let $\mu_{0}$ be any initial value of $\mu$, and the process of Gibbs sampling is as follows:

$$
\begin{aligned}
& \text { Step 1: } k_{1} \operatorname{from} f_{1}(k \mid \mu, \mathrm{X}, \mathrm{M}) \\
& \text { Step 2: } \mu_{1} \operatorname{from} f_{2}\left(\mu \mid k_{1}, \mathrm{X}, \mathrm{M}\right)
\end{aligned}
$$

This completes a Gibbs iteration, and the parameters change to $k_{1}$ and $\mu_{1}$. Using the new parameter as the initial value, repeat the previous random sampling iteration, thus completing another Gibbs iteration process. After repeating for $m$ times, a series of random samples are obtained: $\left(\mu_{1}, k_{1}\right), \ldots,\left(\mu_{m}, k_{m}\right)$.

By repeating $n$ large enough iterations and discarding the previous $m$ random samples, Gibbs samples will be obtained: $\left(\mu_{m+1}, k_{m+1}\right), \ldots,\left(\mu_{n}, k_{n}\right)$.

\section{Full-Scale Test for Friction Coefficients}

4.1. Test Design. In order to study the friction characteristics of prestressing loss, four prefabricated box girders with span of $20 \mathrm{~m}, 25 \mathrm{~m}$, and $30 \mathrm{~m}$ (two box girders) were selected as test beams. The strength design grade of structural concrete was C50. The 15.2-1860 standard prestressing strands were adopted as steel strands, whose tension control stress under anchor and outside anchor was $1350 \mathrm{MPa}$ and1437 $\mathrm{MPa}$, respectively, and the elastic modulus of prestressing tendon was $\mathrm{Ep}=1.95 * 10^{5} \mathrm{MPa}$. The prestressing force was symmetrically distributed along the vertical central line of the cross section of the beam.

Four beams were chosen as samples for the full-scale field tests to monitor tendons. Two web bundles (N1 and $\mathrm{N} 3$ ) and one floor bundle (N4 for $20 \mathrm{~m}$ and N5 for $25 \mathrm{~m}$ and $30 \mathrm{~m}$ ) were deployed with sensors (anchor cable dynamometer and EM sensors) in each test beam. The cross sections of prestressed reinforcement distribution in the field test beam are described in Figure 1.
4.2. Layout of Measuring Points. The anchor cable dynamometers were embedded in the anchorage end of each test prestressing tendon so as to monitor the true stress under anchorage, while the electromagnetic sensors were embedded in different positions of each test prestressing tendon. Figure 2 outlines the layout of measuring points, and the parameters ( $a$ to $h$ ) of diverse test beams are shown in Table 2.

4.3. Anchor Cable Dynamometer. The anchor cable dynamometer is a kind of piercing multistring pressure sensor, which can be used for cable force measurement and construction control during cable tension. Moreover, cable dynamometer may be applied for Jack force calibration when tensioning prestressing steel bar. Apart from that, it is well-adapted to long-term monitoring and automatic monitoring. Cable dynamometer is an intelligent temperature type, measuring results of which are calibrated according to the measured temperature. Six-string intelligent anchor cable dynamometer is used in this test, as shown in Figure 3.

4.4. Electromagnetic (EM) Sensor. The electromagnetic sensor is mainly composed of primary and secondary coils, where a temperature sensor is built-in for temperature compensation. Figure 4 indicates the structure sketch of the electromagnetic sensor. When the primary coil passes into the pulse current, the secondary coil generates instantaneous current, thus obtaining the induced voltage. The induced voltage magnitude depends on the permeability of the specimen, which may exert an influence on the stress of the specimen. Therefore, the relationship between the induced voltage and the stress can be measured. Electromagnetic sensor was applied to PSC girders, and the sensor and reader are shown in Figure 5.

\section{Results and Discussion}

The experiments obtained values as parameters from equation (2) are shown in Table 3, where the value of tension control stress is $f_{p j}$, the value of friction loss stress is $\Delta f_{p F}$, the value of distance is $x$, and the value of sag ratio is $\theta$. Twenty sets of data of the first three beams (beam $=20 \mathrm{~m}, 25 \mathrm{~m}$, $30 \mathrm{~m}$ ) were regressed by least square method and Bayesian quantile, respectively, and the parameters were tested by five sets of data of the fourth beam.

Figure 6 only reveals the sample traces of the estimated parameters at the quantile of 0.5 because they can represent the rest of figure. A standard normal distribution was selected as its prior distribution for the estimated parameters [33], and the Gibbs sampling algorithm was adopted. To ensure that the chain was stable and the results were more accurate, the number of iterations was set to 12000 .

In order to check the convergence of the MCMC chain, the posterior distribution of the edges can be visually displayed by drawing a histogram of the sampling parameters ( $\mu$ and $k$ ). The histograms of the posterior distribution of regression parameters are overlaid with the density of the 


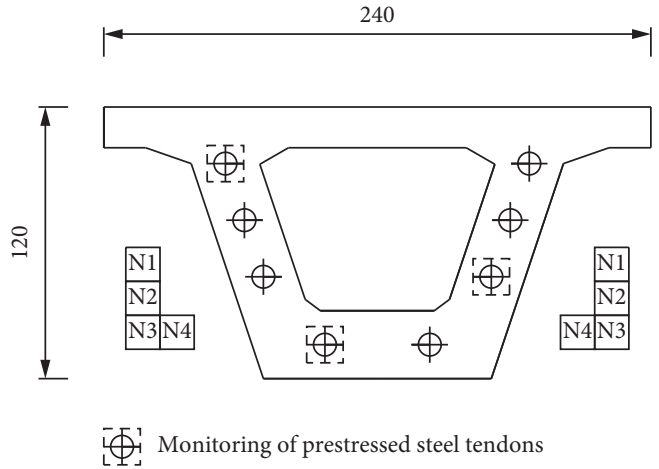

(a)

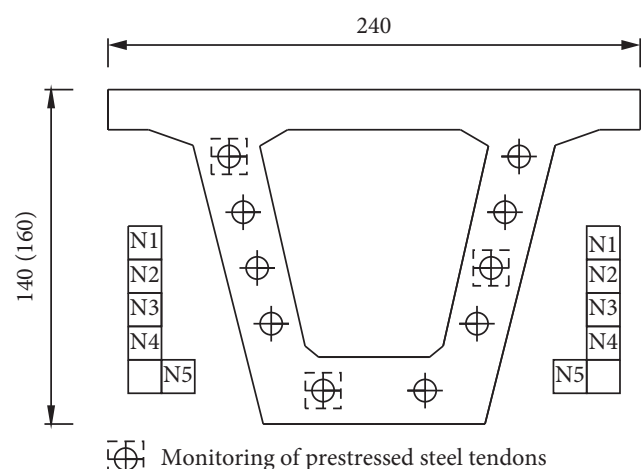

(b)

FiguRE 1: Monitoring of prestressed steel tendons: (a) $20 \mathrm{~m}$ box girder; (b) $25 \mathrm{~m}$ (30 m) box girder (unit: $\mathrm{cm}$ ).

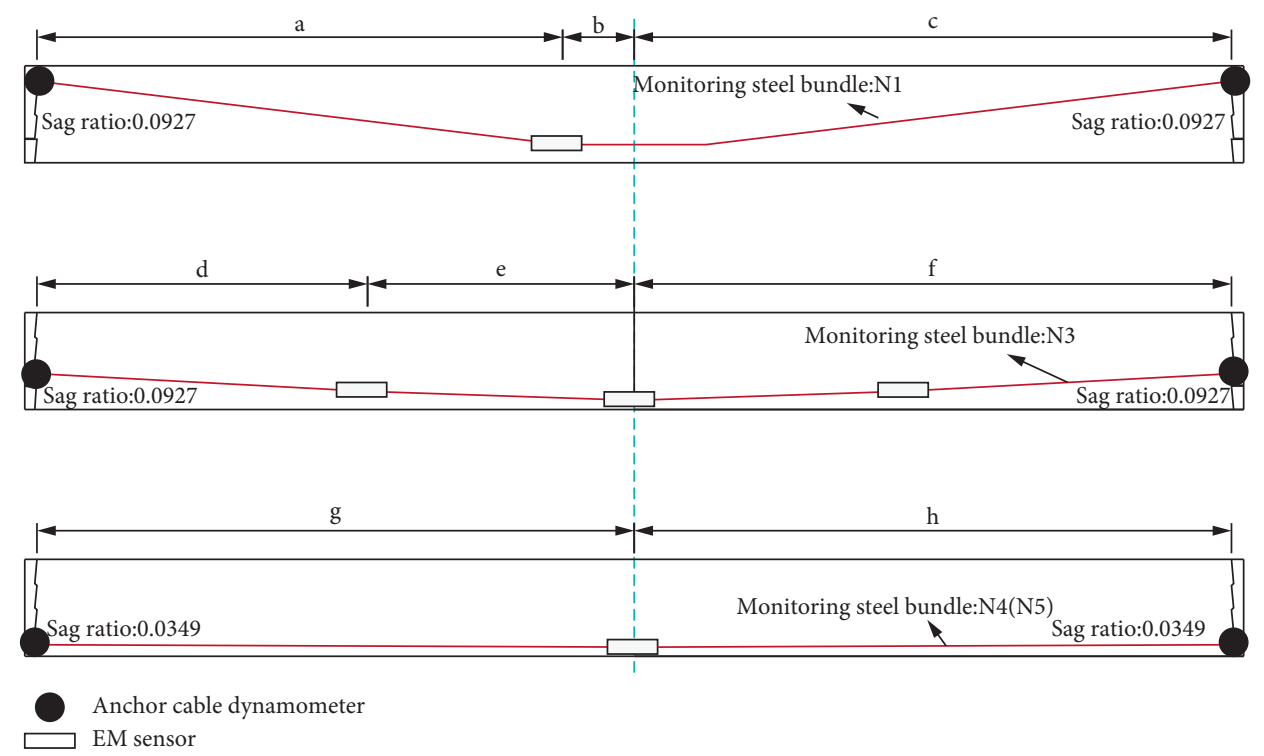

FIGURE 2: Distribution of prestressed monitoring points of single test beam in the process of tension.

TABLe 2: The values of various parameters in the prestress distribution diagram (unit: $\mathrm{mm}$ ).

\begin{tabular}{|c|c|c|c|c|c|c|c|c|}
\hline \multirow{3}{*}{$\begin{array}{l}\text { Beams } \\
(\mathrm{m})\end{array}$} & \multicolumn{8}{|c|}{ Parameter } \\
\hline & \multicolumn{3}{|c|}{ N1 } & \multicolumn{3}{|c|}{ N3 } & \multicolumn{2}{|c|}{ N4 (N5) } \\
\hline & $\mathrm{a}$ & $\mathrm{b}$ & c & $\mathrm{d}$ & $\mathrm{e}$ & $\mathrm{f}$ & g & $\mathrm{h}$ \\
\hline 20 & 8625 & 1175 & $\begin{array}{c}19600 / \\
2\end{array}$ & 5425 & 4375 & $\begin{array}{c}19600 / \\
2\end{array}$ & 9800 & $\begin{array}{c}19600 / \\
2\end{array}$ \\
\hline 25 & 9874 & 2426 & $\begin{array}{c}24600 / \\
2\end{array}$ & 6453 & 5847 & $\begin{array}{c}24600 / \\
2\end{array}$ & 12300 & $\begin{array}{c}24600 / \\
2\end{array}$ \\
\hline 30 & 12080 & 2720 & $\begin{array}{c}29600 / \\
2\end{array}$ & 7223 & 5577 & $\begin{array}{c}29600 / \\
2\end{array}$ & 14800 & $\begin{array}{c}29600 / \\
2\end{array}$ \\
\hline 30 & 12080 & 2720 & $\begin{array}{c}29600 / \\
2\end{array}$ & 7223 & 5577 & $\begin{array}{c}29600 / \\
2\end{array}$ & 14800 & $\begin{array}{c}29600 / \\
2\end{array}$ \\
\hline
\end{tabular}

normal approximation of the posterior distribution as shown in Figure 7. Two thousand draws were excluded as burn-in draws, aiming to make sure only draws from the stationary posterior distribution are plotted.
The Bayesian quantile estimates and least squares estimates of the estimated parameters at different quantiles $(p=0.05,0.25,0.5,0.75,0.95)$ are listed in Table 4 . And the simulations were performed on the programming package $R$ software.

Figure 8 gives information of the quantile plots for the $k$ and $\mu$ variables, plotted according to data in Table 4 . There is a negative impact when $\mu$ is below the quantile of 0.25 whereas a positive impact when $\mu$ is above the quantile of 0.25 , and this effect becomes more pronounced for higher quantiles. And the estimated value obtained by the OLS method is larger than the Bayesian median. The expected value of $\mu$ for the overall quantile is dramatically lower than the mean value. The variable $k$ responds insignificantly to the expected median, but the quantile plot shows that it has a negative effect on lower quantiles and a positive effect on higher quantiles, indicating that $k$ should not be ignored. And both the estimated value calculated by the OLS method and Bayesian median are 


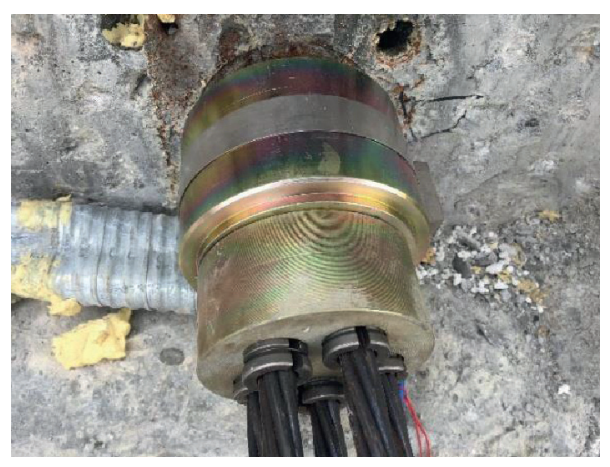

(a)

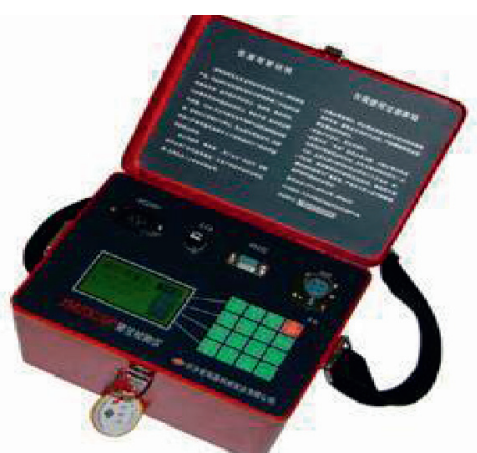

(b)

FIgURE 3: The anchor cable dynamometer: (a) application of electromagnetic sensor to PSC girders; (b) the anchor cable dynamometer reader.

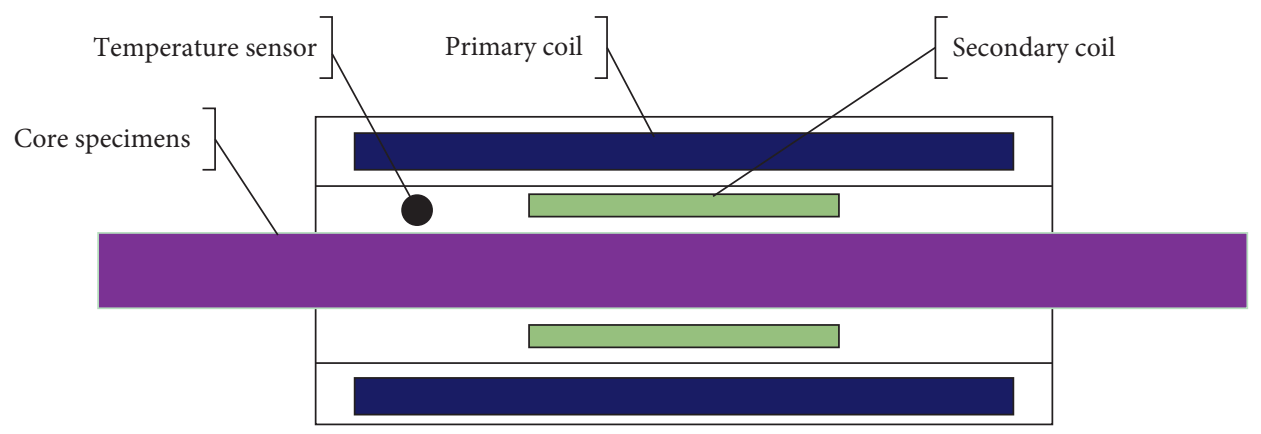

FIGURE 4: Structural sketch of electromagnetic (EM) sensor.

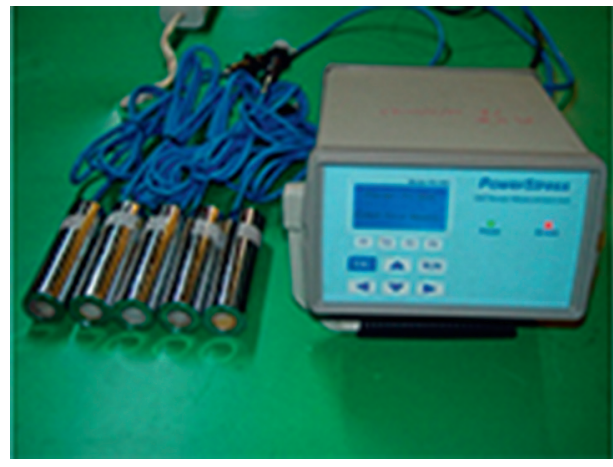

(a)

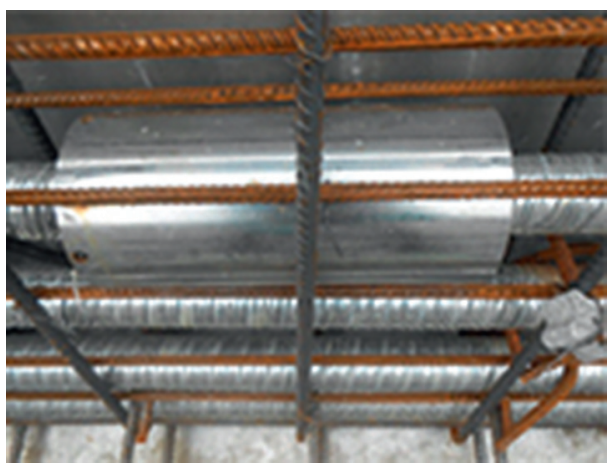

(b)

FIGURE 5: (a) Electromagnetic sensor and reader; (b) application of electromagnetic sensor to PSC girders.

steadily close to zero, which means that, for the average of the predicted values, the expected value of $k$ in the lower quantile is greatly lower than the average.

Figure 9 demonstrates the range of friction coefficients in the four codes and the friction coefficients obtained by regression. The results show that the friction coefficients obtained in the actual PSC girder bridge are different from those determined in the tests, and the full-scale tests contain many uncertainties. Specifically, all of the regulations of curvature friction coefficient values in the specifications are range values. The curvature friction coefficient values obtained by the least square method are larger than those given by the four specifications, while the $\mu$ values obtained by Bayesian quantile regression approach the lower limit in JTG 3362-2018, in the range given by ACI 318-08, ACI 318-14, and AASHTO 
TABLE 3: Full-scale field test measured values.

\begin{tabular}{|c|c|c|c|c|c|}
\hline Beam $(\mathrm{m})$ & Number of steel & Tension control stress $(\mathrm{MPa})$ & Distance $(\mathrm{m})$ & Sag ratio (rad) & Friction loss stress (MPa) \\
\hline \multirow{5}{*}{20} & \multirow{2}{*}{ N1 } & 1337.86 & 8.625 & 0.0927 & 87.32 \\
\hline & & 1499.29 & 5.425 & 0.0927 & 79.29 \\
\hline & \multirow{2}{*}{ N3 } & 1499.29 & 9.800 & 0.0927 & 102.86 \\
\hline & & 1499.29 & 5.425 & 0.0927 & 85.54 \\
\hline & $\mathrm{N} 4$ & 1336.14 & 9.800 & 0.0349 & 65.14 \\
\hline \multirow{5}{*}{25} & \multirow{2}{*}{ N1 } & 1356.07 & 9.654 & 0.0927 & 92.68 \\
\hline & & 1315.71 & 6.453 & 0.0927 & 55.00 \\
\hline & \multirow{2}{*}{ N3 } & 1315.71 & 12.300 & 0.0927 & 130.14 \\
\hline & & 1229.57 & 6.453 & 0.0927 & 60.57 \\
\hline & N5 & 1232.71 & 12.300 & 0.0349 & 75.86 \\
\hline \multirow{5}{*}{30} & \multirow{2}{*}{ N1 } & 1427.5 & 12.080 & 0.0927 & 77.62 \\
\hline & & 1347.86 & 9.233 & 0.0927 & 58.57 \\
\hline & \multirow{2}{*}{$\mathrm{N} 3$} & 1347.86 & 14.80 & 0.0927 & 79.4 \\
\hline & & 1273.93 & 9.233 & 0.0927 & 96.67 \\
\hline & N5 & 1335.57 & 14.800 & 0.0349 & 98.67 \\
\hline \multirow{5}{*}{30} & \multirow{2}{*}{ N1 } & 1285.43 & 12.080 & 0.0927 & 69.14 \\
\hline & & 1404.52 & 9.233 & 0.0927 & 64.17 \\
\hline & \multirow{2}{*}{ N3 } & 1404.52 & 14.80 & 0.0927 & 82.86 \\
\hline & & 1283.81 & 9.233 & 0.0927 & 92.14 \\
\hline & N5 & 1311.00 & 14.800 & 0.0349 & 88.86 \\
\hline
\end{tabular}

(1) Distance and sag ratio are known values, and tension control stress and friction loss stress are measured values. (2) Measured values are measured in the final stage of tension.

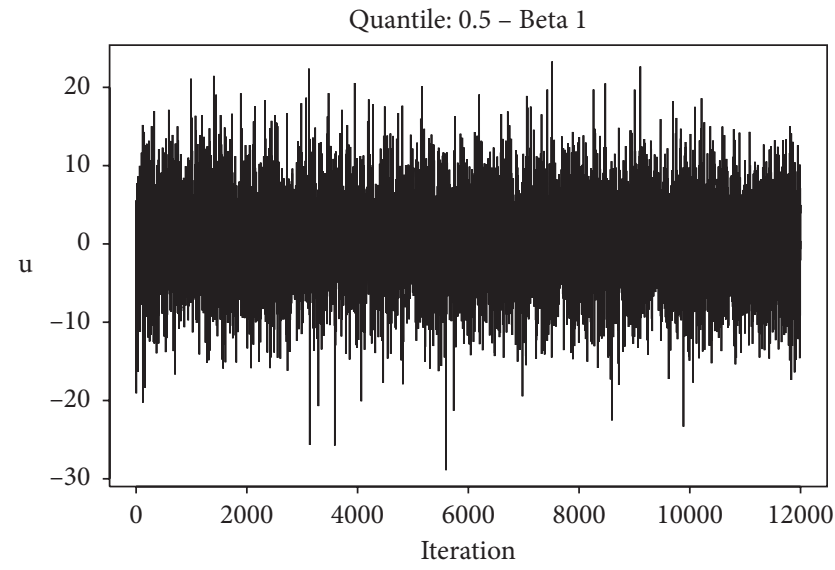

(a)

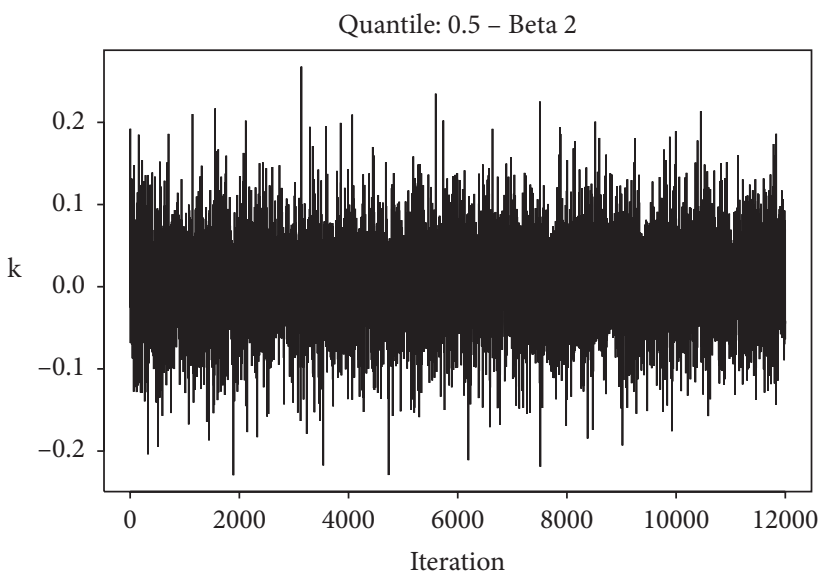

(b)

FIgURE 6: Traceplots of the MCMC chains for quantile regression: (a) curvature friction coefficient $\mu$; (b) wobble friction coefficient (k).

specifications. On the other hand, the wobble friction coefficient values obtained by both methods are in the middle of ACI 318-08 [13]. To be more exact, the wobble friction coefficient values obtained by Bayesian regression are larger than those obtained by least squares method, closer to the middle, but still larger than those specified in the other three specifications. Generally speaking, the values given by ACI 318-08 are relatively conservative and have a wider range for the friction coefficient. The friction coefficients obtained by different calculation methods are quite different, so it is difficult to give a single fixed value.
The last five sets of data in Table 3 were used to test the sample mean hypothesis of the regression parameters obtained by the two methods. The test statistics of the two methods were compared with each other, and the results are shown in Table 5.

From the data in the table, under the significance level of $\alpha=0.05$, there was no obvious difference between Bayesian quantile regression predicted value and test value, whilst the least squares predicted value and test value presented an enormous difference. Therefore, the Bayesian quantile regression parameters are more reasonable than the least squares regression parameters. 


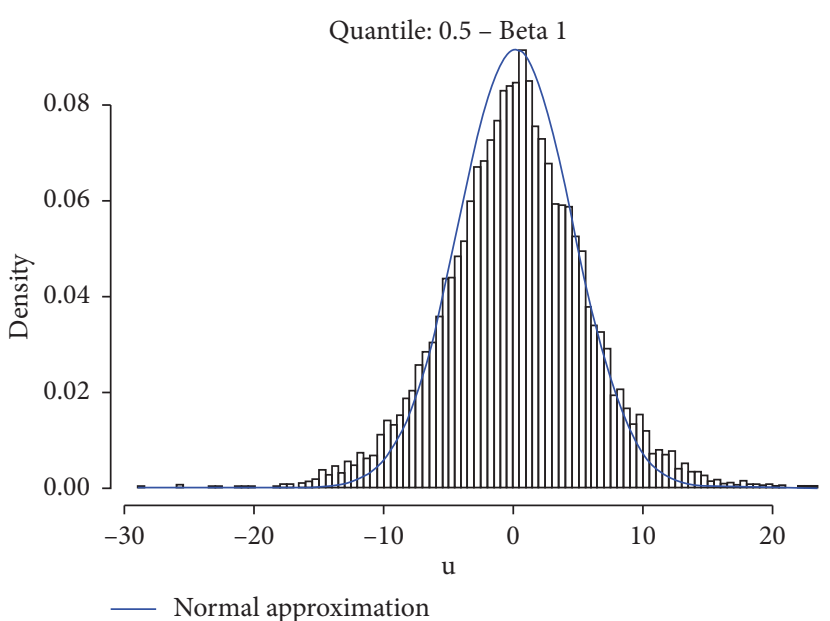

(a)

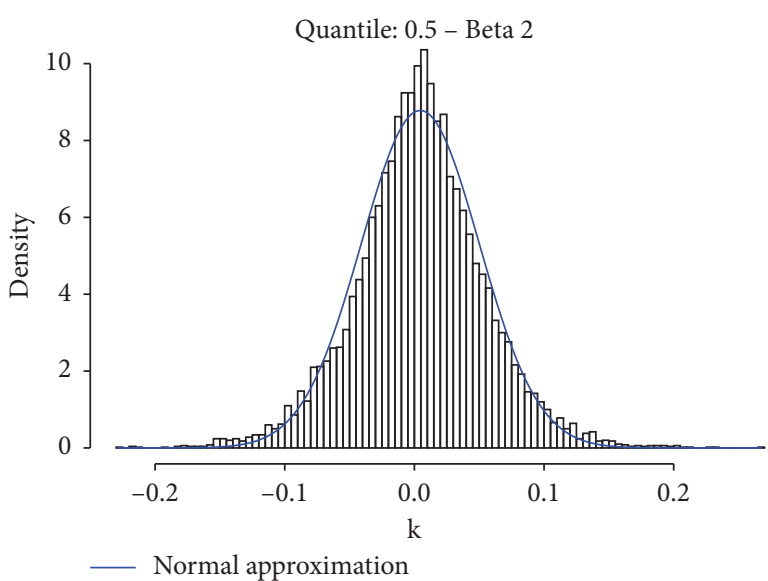

(b)

Figure 7: Posterior histograms for quantile regression: (a) curvature friction coefficient $\mu$; (b) wobble friction coefficient (k).

TABLE 4: Summary of friction coefficients.

\begin{tabular}{lccc}
\hline Friction coefficients & $p$ & Bayes estimate & OLS \\
\hline \multirow{4}{*}{$\mu$} & 0.05 & -0.159 & \\
& 0.25 & -0.001 & 0.308 \\
& 0.5 & 0.197 & \\
& 0.75 & 0.385 & \\
& 0.95 & 0.538 & 0.004 \\
& 0.05 & -0.152 & \\
& 0.25 & -0.020 & 0.005 \\
\end{tabular}

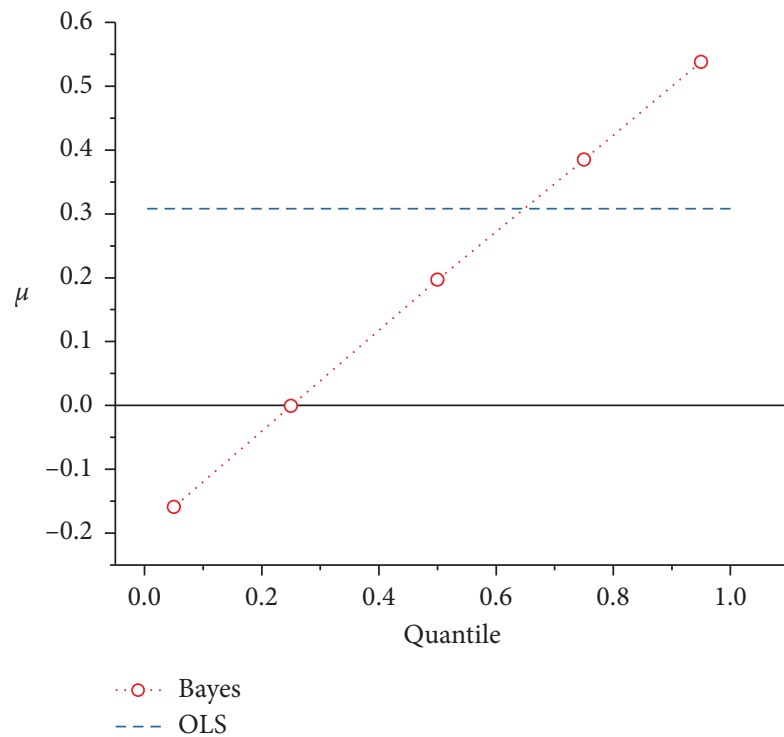

(a)

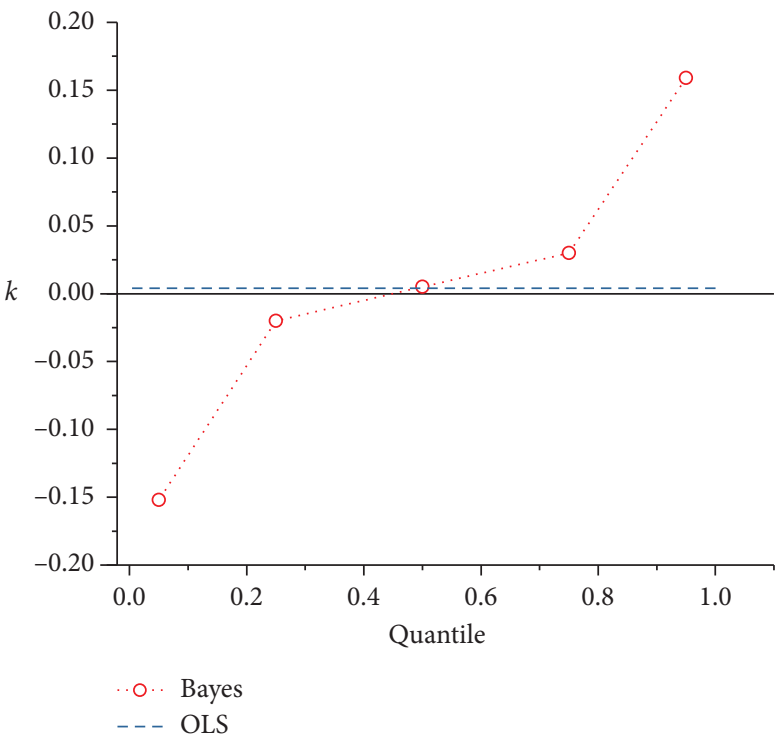

(b)

FIGURE 8: Quantile plots for the (a) curvature friction coefficient and (b) wobble friction coefficient. Dotted lines represent the OLS estimate. 


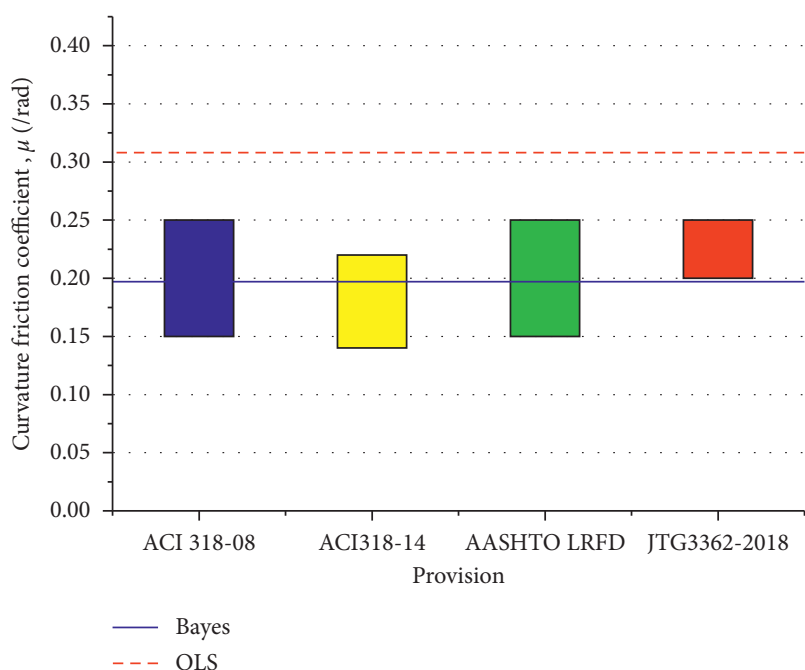

(a)

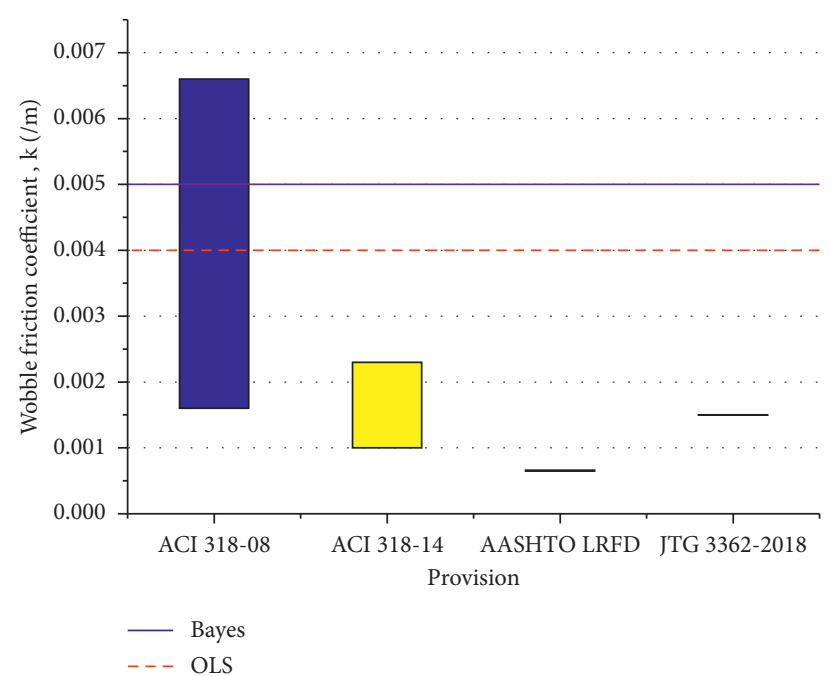

(b)

Figure 9: Comparison of range of friction coefficients: (a) curvature friction coefficient $(\mu)$ and (b) wobble friction coefficient (k). The histograms represent the recommended parameter value ranges given in the specifications.

TABle 5: Parametric hypothesis testing.

\begin{tabular}{lcc}
\hline Bayes estimate & OLS & $t_{\alpha / 2}$ \\
\hline 2.310 & 2.660 & 2.447 \\
\hline
\end{tabular}

\section{Conclusion}

Four prefabricated prestressed box girder bridges with spans of 20,25, and 30 meters were adopted for full-scale field tests, and the friction coefficient was investigated, which was very rare in previous relevant studies. The least square method and Bayesian quantile regression method were used to analyze the actual friction coefficient data sets of real bridges. Field test results indicated that there was a significant difference between the prediction value and the test value of the least square method. Nevertheless, the predicted value and the test value of Bayesian quantile regression showed no obvious difference. The value of curvature friction coefficient obtained by least squares regression was $0.308 / \mathrm{rad}$, larger than the specified value, so it was not adopted. However, the value of curvature friction coefficient obtained by Bayesian quantile regression was $0.197 / \mathrm{rad}$, which was similar to the median value recommended by ACI 318-08 and could be adopted. The wobble friction coefficient values obtained by regression of the two methods were $0.004 / \mathrm{m}$ and $0.005 / \mathrm{m}$, respectively. Besides, the regression values were within the scope of ACI 318-08 and were larger than those recommended in other specifications. As a consequence, in the practical engineering, when the number of samples is small, the Bayesian quantile algorithm proposed in this paper should be used to obtain the friction coefficient for the purpose of higher accuracy.

\section{Data Availability}

The data supporting the conclusions of the study can be obtained in the manuscript.

\section{Conflicts of Interest}

The authors declare that there are no conflicts of interest regarding the publication of this paper.

\section{Acknowledgments}

The authors would like to acknowledge the financial support from Chinese Universities Scientific Fund no. 300102218516 and Guangdong Provincial Transportation and Transportation Office Technology Funding Project (2016-02-016).

\section{References}

[1] China Communications press Co., Ltd., Specifications For Design Of Highway Reinforced Concrete and Prestressed Concrete Bridges And Culverts, China Communications press Co., Ltd., Beijing,China, 2018.

[2] A. Committee and I. O. F. Standardization, Building Code Requirements for Structural Concrete (ACI 318-14) and Commentary, American Concrete Institute, Farmington Hills, MA, USA, 2014.

[3] AASHTO, AASHTO LRFD Bridge Design Specifications, AASHTO, Washington, DC, USA, 8th edition, 2017.

[4] PTI, Post-tensioning Manual, PTI, Glendale, AZ, US, 6th edition, 2006.

[5] Y. Chen, P. Sareh, J. Feng, and Q. Sun, "A computational method for automated detection of engineering structures with cyclic symmetries," Computers \& Structures, vol. 191, pp. 153-164, 2017.

[6] C.-C. Chou and J.-H. Chen, "Tests and analyses of a full-scale post-tensioned RCS frame subassembly," Journal of Constructional Steel Research, vol. 66, no. 11, pp. 1354-1365, 2010.

[7] Y. Chen, Q. Zhang, J. Feng et al., "Experimental study on shear resistance of precast RC shear walls with novel bundled connections," Journal of Earthquake and Tsunami, vol. 13, no. 3-4, Article ID 1940002, 2019. 
[8] A. C. Aparicio, G. Ramos, and J. R. Casas, "Testing of externally prestressed concrete beams," Engineering Structures, vol. 24, no. 1, pp. 73-84, 2002.

[9] S.-J. Jeon, S. Y. Park, S.-H. Kim, S. T. Kim, and Y. Park, "Estimation of friction coefficient using smart strand," International Journal of Concrete Structures and Materials, vol. 9, no. 3, pp. 369-379, 2015.

[10] S.-H. Kim, S. Y. Park, Y. Park, and S.-J. Jeon, "Friction characteristics of post-tensioning tendons in full-scale structures," Engineering Structures, vol. 183, pp. 389-397, 2019.

[11] Y. Zhang, S. Meng, Z. Xi et al., "Experimental research on prestressing time-dependent loss of high-performance concrete beams," Industrial Construction, vol. 39, no. 12, pp. 21-24, 2009.

[12] S. Kurokawa, S. Sumitro, K. Shimano et al., "Long term stress monitoring on PC beam by using elasto-magnetic sensor," The First Fib Congress, Concrete Structures in the 21th Century, pp. 13-19, 2002.

[13] T. Dedong, H. Shanglian, and C. Weimin, "Research of cable tension sensor based on magneto-elastic effect in cable-stayed bridge," Transducer and Microsystem Technology, vol. 25, no. 10, pp. 28-30, 2006.

[14] L. Ren, X. Yao, C. Xiu et al., "Experimental study on optimization of elasto-magnetic (EM) sensor performance based on ratio measurement," Chinese Journal of Sensors and Actuators, vol. 32, no. 5, pp. 658-662, 2019.

[15] P. J. S. P. Gupta, "Rational determination of friction losses in post-tensioned construction," ACI Symposium Publication, vol. 231, pp. 129-144, 2005.

[16] S. J. Jeon, 박종칠, I. K. Park et al., "Estimation of friction coefficients based on field data," Journal of the Korean Society of Civil Engineers A, vol. 29, no. 5, pp. 487-494, 2009.

[17] T. Kitani and A. Shimizu, "Friction coefficient measurement test on $13 \mathrm{MN}$ class tendon of PC strands for prestressed concrete containment vessel (PCCV)(6-1825)," in Proceedings of the 20 th International Conference on Structural Mechanics in Reactor Technology, p. 206, Espoo, Finland, August, 2009.

[18] E. Kreyszig, Advanced Engineering Mathematics, Wiley, Hoboken, NJ, USA, 10, 2011.

[19] K. Yu and R. A. Moyeed, "Bayesian quantile regression," Statistics \& Probability Letters, vol. 54, no. 4, pp. 437-447, 2001.

[20] K. Yu, Z. Lu, and J. Stander, "Quantile regression: applications and current research areas," Journal of the Royal Statistical Society: Series D (The Statistician), vol. 52, no. 3, pp. 331-350, 2003.

[21] A. Committee and I. O. f. Standardization, Building Code Requirements for Structural Concrete (ACI 318-08) and Commentary, American Concrete Institute, Farmington Hills, Michigan, US, 2008.

[22] PCI, Bridge Design Manual, MNL, PCI, IL, US, 3rd edition, 2011.

[23] B. J. G. Koenker R, "Regression quantiles," Journal of the Econometric Society, vol. 46, no. 46, pp. 33-50, 1978.

[24] R. Koenker and G. Bassett, "Robust tests for heteroscedasticity based on regression quantiles," Econometrica, vol. 50, no. 1, pp. 43-61, 1982.

[25] M. Buchinsky, "Estimating the asymptotic covariance matrix for quantile regression models a Monte Carlo study," Journal of Econometrics, vol. 68, no. 2, pp. 303-338, 1995.

[26] V. Chernozhukov, C. Hansen, and M. Jansson, "Finite sample inference for quantile regression models," Journal of Econometrics, vol. 152, no. 2, pp. 93-103, 2009.
[27] R. Koenker, Quantile Regression, Cambridge University Press, London, UK, 2005.

[28] T. Kozubowski and K. Podgorski, "Asymmetric Laplace distributions," The Mathematical Scientist, vol. 25, pp. 37-46, 2000.

[29] K. Yu, P. Van Kerm, and J. Zhang, "Bayesian quantile regression: an application to the wage distribution in 1990s Britain," Sankhya Ser A, vol. 67, 2004.

[30] E. G. Tsionas, "Bayesian quantile inference," Journal of Statistical Computation and Simulation, vol. 73, no. 9, pp. 659674, 2003.

[31] T. Lancaster and S. Jae Jun, "Bayesian quantile regression methods," Journal of Applied Econometrics, vol. 25, no. 2, pp. 287-307, 2010.

[32] D. F. Benoit and D. V. D. Poel, "Binary quantile regression a bayesian approach based on the asymmetric laplace density," Ghent University, Faculty of Economics and Business Administration, 2010.

[33] S. T. Tokdar and J. B. Kadane, "Simultaneous linear quantile regression: a semiparametric bayesian approach," Bayesian Analysis, vol. 7, no. 1, pp. 51-72, 2012.

[34] D. F. Benoit, D. van den Poel, and Q. R. Bayes, "A bayesian approach to quantile regression," Journal of Statistical Software, vol. 76, no. 7, pp. 1-32, 2017.

[35] P. Das and S. Ghosal, "Bayesian quantile regression using random B-spline series prior," Computational Statistics \& Data Analysis, vol. 109, pp. 121-143, 2017.

[36] M. Lubrano and A. Ndoye, "Bayesian unconditional quantile regression: an analysis of recent expansions in wage structure and earnings inequality in the US 1992-2009," Scottish Journal of Political Economy, vol. 61, 2014.

[37] A. X. D. Dong, J. S. K. Chan, and G. W. Peters, "Risk margin quantile function via parametric and non-parametric bayesian approaches," ASTIN Bulletin, vol. 45, no. 3, pp. 503-550, 2015.

[38] M. A. Tanner and W. H. Wong, "The calculation of posterior distributions by data augmentation," Journal of the American Statistical Association, vol. 82, no. 398, pp. 528-540, 1987.

[39] A. E. Gelfand and A. F. M. Smith, "Sampling-based approaches to calculating marginal densities," Journal of the American Statistical Association, vol. 85, no. 410, pp. 398-409, 1990.

[40] J. Besag, P. Green, D. Higdon, and K. Mengersen, "Bayesian computation and stochastic systems," Statistical Science, vol. 10, no. 1, pp. 3-41, 1995.

[41] X. Wei, D. Xiao-Xia, and F. J. C. P. B. Yuan, "BayesianMCMC-based parameter estimation of stealth aircraft RCS models," Chinese Physics B, vol. 24, no. 12, p. 129501, 2015.

[42] H. Kozumi and G. Kobayashi, "Gibbs sampling methods for Bayesian quantile regression," Journal of Statistical Computation and Simulation, vol. 81, no. 11, pp. 1565-1578, 2011. 